\title{
A Note on the Path Integral Representation of the Boundary State of D-brane
}

\author{
Yong Zhang* \\ Institute of Theoretical Physics, Academia Sinica, \\ PO Box 2735, Beijing 100080, P. R. China
}

\begin{abstract}
In this paper we construct path integral representations of the boundary states in some special backgrounds such as the $U(1)$ gauge field background, the linear dilaton background and the open string tachyon background. The initial purpose of this paper is to construct a general solution of the boundary conformal field theory with the analytical approach, mainly for the constraint equations $\left(L_{n}-\tilde{L}_{-n}\right)|B\rangle=0$ are difficult to be solved to obtain the solution represented by string modes from the pure algebraic approach. However in the path integral representation it is easy transforming those algebraic equations into the differential equations which can be solved. Another purpose of this paper is to try to explore an open question. we do not know how to construct an exact theory of D-branes in the general background until now. However many recent researches show the boundary state description indeed seizes some fundamental features of D-branes in the rather special backgrounds. Since the general background field effects can be easily introduced in the path integral representation, we argue that path integral representation of the boundary state should provide an exact description of $\mathrm{D}$ branes in the general backgrounds.
\end{abstract}

\footnotetext{
${ }^{*}$ zhangyo@itp.ac.cn
} 


\section{Contents}

1. Introduction

2. The path integral representation of the boundary state in the $U(1)$ gauge field background

3

2.1 The path integral representation of the boundary state

2.2 T-duality of D-brane

2.3 The path integral representation of the boundary state in the $U(1)$ gauge

field background

2.4 Some notes of the path integral representation of the boundary state

3. The path integral representation of the boundary state in other special backgrounds

3.1 The path integral representation of the boundary state in the linear dilaton background

3.2 The path integral representation of the boundary state in the open string tachyon background

4. Discussions

15

A. The calculation of the normalization factor $N(F)$

B. The conformal path integral representation of the boundary state in the linear dilaton background

\section{Introduction}

It is well known that D-branes in 11 play very important roles in the study of nonperturbative string theory dynamics. However, we do not know how to construct an exact theory of D-brane in the general background until now. So we have to limit ourselves within a very small circle, namely in some special backgrounds. This kind of study may have some heuristic implications in seeking a good theory describing D-brane. On the other hand many recent researches show the boundary state description indeed 
seizes some basic features of D-brane. In fact the concept of the boundary state is introduced into string theory before that of D-brane. The BPS object can be represented by boundary state, which satisfies the constraint $(Q+\tilde{Q})|B\rangle=0$. We can read off the tension and R-R charge from the tree diagram of the boundary state. These are the most important proofs supporting the conjecture that D-brane is the classical soliton solution with R-R charge, namely p-brane. Many relevant literatures have been included in [2].

The concept of the boundary state is fundamental. Firstly, the tadpole can be represented with the boundary state which is the source of closed string. Naively, it seems reasonable that the boundary state should be seen as quantum state of an independent dynamic object arising from string vacuum. D-brane is just defined as that object where open string end points end. Secondly, the boundary state can be defined according to the idea that one loop amplitude of open string can be regarded as tree amplitude of closed string. From this physical picture, the holographic principle [3] can be argued because non-abelian gauge field theory appear from the low energy limits of open string theory and the low energy limit of closed string theory can lead to effective theory of gravity. Thirdly in [4] Cardy generalizes the boundary state concept so as to get an exact description of the boundary conformal field theory. Now the boundary conformal field theory of Gepner model, which is important in the heterotic string theory, has been proposed in [5]. This generalizes D-brane concept, since the original D-brane only comes from the system of Type I string, Type IIA string and Type IIB string. Fourthly, in [6], the path integral representation of boundary state provides a simple proof supporting the fundamental argument in Matrix theory that physics of $D_{p}$ brane is equivalent to that of infinitely many $D_{(p-2 r)}$ branes. Fifthly, the path integral formalism has been incorporated into recent development of Noncommutative geometry in string theory in [7]. Finally, in [2] the classical $p$-brane solution can be constructed from one point amplitude of boundary state with closed string states. This is also an important proof that D-brane is the classical soliton solution with $\mathrm{R}$ - $\mathrm{R}$ charge.

Here we only give some examples of the path integral representation of the conformal invariant boundary state ${ }^{1}$. Since the boundary conformal field theory is a candidate for D-brane theory, we must find solutions of the boundary conformal field theory. In the case of path integral representation, algebraic constraint equation can be transformed into differential equation which is easily solved to obtain the solution represented by string modes. Although the conformal invariant boundary state satis-

\footnotetext{
${ }^{1}$ The Feynman path integral is one way to represent a quantum theory, and it is a very natural method for describing interactions in string theory. Since we have had a systematic study of string theory using the Polyakov path integral, we may try to explore the path integral representation of D-brane.
} 
fying Cardy condition can be constructed with Ishibashi state, this formalism is not often used in the practical calculation. For example in those review articles [2] the mode representation of the boundary state is used calculating various amplitudes ${ }^{2}$.

The organization of this paper is as follows. In section 2, only from conformal invariance we deduce the path integral representation of boundary state in constant $U(1)$ gauge field background ${ }^{3}$. and show some applications of this formalism in Tduality and Matrix theory. In section 3, we construct the conformal invariant boundary state in the linear dilaton background with the path integral representation, and at the same time construct the path integral representation of the boundary state in open string tachyon background. Section 4 is devoted to discussions.

\section{The path integral representation of the boundary state in the $U(1)$ gauge field background}

The boundary state must be conformal invariant, which is the requirement of the reparametrization invariance on the world sheet. So the conformal invariance can be directly used to construct the path integral representation of boundary state in the general background. In this section, firstly this procedure will be given in detail. Secondly T-duality of D-brane to construct $D_{p}$ brane from $D_{-1}$ brane can be carried out in the path integral formalism of the boundary state. Then the simplest solution of those differential equations is found right to be the path integral representation of boundary state in constant $U(1)$ gauge field background.

\subsection{The path integral representation of the boundary state}

In this paper only consider the bosonic string case. In fact the generalization to supersymmetrical case is straightforward. The holomorphic stress tensor reads

$$
T=-\frac{1}{2}:(\partial X)^{2}:
$$

\footnotetext{
${ }^{2}$ It is necessary to notice the path integral representation of boundary state is easily connected with divergence. Although the path integral representation of the boundary state in non-constant $U(1)$ gauge field background in [8] can be obtained, those boundary states contain bad divergences in [8] and so need regularization and renormalization. Another example is the path integral representation of the boundary state in the linear dilaton background whose procedure of regularization and renormalization will be discussed in Appendix B.

${ }^{3}$ Although the path integral representation of boundary state in constant $U(1)$ gauge field background in [9] had been argued so as to determine the normalization factor, here the familiar form of path integral representation in [9] can be deduced directly from the conformal invariance.
} 
and a similar anti-holomorphic counterpart. It is necessary to work with mode expansions

$$
\begin{aligned}
X(\tau, \sigma) & =q+\left(\alpha_{0}+\tilde{\alpha}_{0}\right) \tau+i \sum_{n \neq 0} \frac{1}{n}\left(\alpha_{n} e^{-i n(\tau-\sigma)}+\tilde{\alpha}_{n} e^{-i n(\tau+\sigma)}\right), \\
X(z, \bar{z}) & =q-i\left(\alpha_{0} \ln z+\tilde{\alpha}_{0} \ln \bar{z}\right)-i \sum_{n \neq 0} \frac{1}{n}\left(\alpha_{-n} z^{n}+\tilde{\alpha}_{-n} \bar{z}^{n}\right), \\
L_{n} & =\frac{1}{2} \sum_{m}: \alpha_{m+n} \alpha_{-m}:
\end{aligned}
$$

in which we have taken the Regge slope $\alpha^{\prime}=2$. The commutators are $\left[\alpha_{m}, \alpha_{n}\right]=$ $m \delta_{m+n, 0}$, and similarly for the right-moving modes. Let $K_{n}=L_{n}-\tilde{L}_{-n}$. The boundary condition is entirely encoded in the boundary state $|B\rangle$, and the conformal invariant condition is $K_{n}|B\rangle=0$. To solve equations $K_{n}|B, p\rangle=0$, it is essential to adopt the coherent state technique introduced in [9]. Introduce the following coherent state which satisfies

$$
\left(\alpha_{n}-\tilde{\alpha}_{-n}-x_{n}\right)|x, p\rangle=0
$$

where $n$ can be either positive or negative. Further the conjugate coherent state should satisfy

$$
\langle x, p|\left(\alpha_{n}-\tilde{\alpha}_{-n}-x_{n}\right)=0 .
$$

If requiring $\langle x, p|=(|x, p\rangle)^{\dagger}$, then $x_{-n}=\left(x_{n}\right)^{\dagger}$. Solve this equation (2.5), the correspondent solution is

$$
|x, p\rangle=\exp \left(\sum_{n=1}^{\infty} \frac{1}{n}\left[-\frac{1}{2} x_{n} x_{-n}+\alpha_{-n} \tilde{\alpha}_{-n}+x_{n} \alpha_{-n}-x_{-n} \tilde{\alpha}_{-n}\right]\right)|p\rangle .
$$

This set of states form a complete orthogonal basis as can be checked. The formulas $e^{A+B}=e^{A} e^{B} e^{\frac{-1}{2}[A, B]}$, and $e^{A} e^{B}=e^{B} e^{A} e^{[A, B]}$ when $[[A, B], A]=[[A, B], B]=0$ are very helpful, and will be often used in the involved calculation in this paper. Observing this coherent state, we can find each oscillation mode can be traced in this state! Basing on this point, our goal may be realized that transforming the algebraic equations into the differential equations. So the detailed operation is in essence a kind of substitution

$$
\begin{gathered}
\alpha_{-n}|x, p\rangle=\left(n \frac{\partial}{\partial x_{n}}+\frac{1}{2} x_{-n}\right)|x, p\rangle, \\
\tilde{\alpha}_{-n}|x, p\rangle=\left(-n \frac{\partial}{\partial x_{-n}}-\frac{1}{2} x_{n}\right)|x, p\rangle,
\end{gathered}
$$


with the requirement of $n \neq 0$. We postulate that the boundary state with the boundary interaction is of the form

$$
|B, p\rangle=\int[d x]|x, p\rangle \exp (S(x))
$$

in which $S(x)$ represents the special boundary interaction. In fact the general form of the boundary interaction should admit string zero mode $\hat{q}$ or differential operator $\frac{\partial}{\partial x}$.

To solve $K_{n}|B, p\rangle=0$, one first computes

$$
K_{n}|x, p\rangle=\left(p x_{n}+\sum_{m \neq 0,-n}^{\infty} m x_{m+n} \frac{\partial}{\partial x_{m}}\right)|x, p=\bar{p}\rangle,
$$

in which $n \neq 0$ is necessary. Substituting the above relations into $K_{n}|B, p\rangle=0$ and integrating by parts, the conformal invariance condition for $S(x)$ is transformed into

$$
\left(-p x_{n}+\sum_{m \neq 0,-n}^{\infty} m x_{m+n} \frac{\partial}{\partial x_{m}}\right) S(x)=0 .
$$

However for $n=0$ case the thing is not similar. Applying the above procedure to

$$
K_{0}|B, p\rangle=0
$$

we obtain the equation

$$
\sum_{m \neq 0}^{\infty} m x_{m} \frac{\partial}{\partial x_{m}} S(x)=0
$$

To our special ansatz of the boundary interaction and the special choice of vacuum state, the solution of the equation (2.13) and the equation (2.11) corresponds to a special conformal invariant boundary state. In order to obtain one boundary state, we may take various ansatz of vacuum state and boundary interaction. For example, if we take vacuum state $|p=-\bar{p}\rangle$, then in the $n \neq 0$ case the equation is the form,

$$
\left(\sum_{m \neq 0}^{\infty} m x_{m+n} \frac{\partial}{\partial x_{m}}\right) S(x)=0
$$

\subsection{T-duality of D-brane}

Since T-duality interchanges Neumann and Dirichlet boundary conditions, a further T-duality in a direction tanget to a Dp-brane reduces it to a $D_{(p-1)}$ brane, while a T-duality in an orthogonal direction turns it into a $D_{(p+1)}$ brane. In the path integral 
representation of the boundary state of D-brane, T-duality of $D_{p}$ brane can be carried out.

The coherent state which satisfies

$$
\left(\alpha_{n}-\tilde{\alpha}_{-n}-x_{n}\right)|x, p=-\bar{p}\rangle=0
$$

has to satisfy the following constraint

$$
\left(\alpha_{n}+\tilde{\alpha}_{-n}\right)|x, p=-\bar{p}\rangle=-2 n \frac{\partial}{\partial x_{-n}}|x, p=-\bar{p}\rangle .
$$

Therefore the boundary state

$$
|B\rangle_{N}=\int[d x]|x, p=-\bar{p}\rangle
$$

satisfies Neumann condition $\partial_{\tau} X(\sigma)|B\rangle_{N}=0$. By carrying out the path integral, its detailed formalism is

$$
|B\rangle_{N}=A \exp \left(\sum_{n=1}^{\infty} \frac{1}{n}\left[-\alpha_{-n} \tilde{\alpha}_{-n}\right]\right)|p=-\bar{p}\rangle
$$

where $\mathrm{A}$ is a proportional constant. Further, another coherent state has to be also defined in order to realize our goal in this subsection. Firstly, some formulas should be given by

$$
\begin{gathered}
X(\sigma)=q+i \sqrt{\frac{\alpha^{\prime}}{2}} \sum_{n \neq 0} \frac{1}{n}\left(\alpha_{n}-\tilde{\alpha}_{-n}\right) e^{i n \sigma}, \\
\partial_{\tau} X(\sigma)=\alpha^{\prime} P+\sqrt{\frac{\alpha^{\prime}}{2}} \sum_{n \neq 0}\left(\alpha_{n}+\tilde{\alpha}_{-n}\right) e^{i n \sigma},
\end{gathered}
$$

where $P$ is the total string momentum. Their commutator is $\left[X(\sigma), P\left(\sigma^{\prime}\right)\right]=i \delta(\sigma-$ $\left.\sigma^{\prime}\right)$, in which the string momentum $P(\sigma)$ at the point labeled by the $\sigma$ is defined as $\frac{1}{2 \pi \alpha^{\prime}} \partial_{\tau} X(\sigma)$. The Fourier expansion of $\delta\left(\sigma-\sigma^{\prime}\right)$ takes the form $\frac{1}{2 \pi} \sum_{m} \exp \left(-i m\left(\sigma-\sigma^{\prime}\right)\right)$. Now define a new coherent state

$$
X(\sigma)|x\rangle=x(\sigma)|x\rangle
$$

The difference between the coherent state (2.5) and (2.21) only lies in that the latter is the position eigenstate $\hat{q}|x\rangle=q|x\rangle$ and the former is the momentum eigenstate $\hat{P}|x, p\rangle=P|x, p\rangle$. Naturally, they may be connected by the transform

$$
\int D x(\sigma)|x\rangle=\int[d x]|x, p=-\bar{p}\rangle
$$


And the coherent state (2.21) can be constructed with the boundary state of instanton namely $D_{-1}$ brane,

$$
|x\rangle=\exp \left(-i \int d \sigma P(\sigma) \cdot x(\sigma)\right)|B\rangle_{-1},
$$

where the boundary state of instanton satisfies the constraint $X(\sigma)|B\rangle_{-1}=0$. We conclude this subsection by constructing $D_{p}$ brane boundary state with $D_{-1}$ brane boundary state

$$
|B\rangle_{p}=\int \prod_{i=0}^{p} D x_{i}(\sigma) \exp \left(-i \int d \sigma P^{i}(\sigma) \cdot x_{i}(\sigma)\right)|B\rangle_{-1}
$$

with $i$ is limited to $i=0,1, \cdots, p^{4}$. It can be checked such $D_{p}$ brane boundary state will satisfy the boundary conditions,

$$
\begin{aligned}
& X^{\mu}(\sigma)|B\rangle_{p}=0, \mu=p+1, \cdots, D-1 \\
& P^{i}(\sigma)|B\rangle_{p}=0, i=0,1, \cdots, p .
\end{aligned}
$$

On the other hand only from the above expression of (2.24), T-duality of boundary state seems to be Fourier transform in the configuration space involved with T-duality.

\subsection{The path integral representation of the boundary state in the $U(1)$ gauge field background}

We now turn to solve the differential equation (2.13) and the differential equation (2.14). It is easy to find one simple solution

$$
S(x)=\frac{1}{4} F_{\mu \nu} \sum_{m \neq 0} \frac{x_{m}{ }^{\mu} x_{-m}^{\nu}}{m}
$$

where $x_{0}=0$ and $F_{\mu \nu}=-F_{\nu \mu}$ are necessary and the factor $\frac{1}{4}$ has been fixed due to the following calculation of the normalization factor. The correspondent boundary state is the form

$$
|B, p=0\rangle=\int[d x] \exp \left(\frac{1}{4} F_{\mu \nu} \sum_{m \neq 0} \frac{x_{m}{ }^{\mu} x_{-m}{ }^{\nu}}{m}\right)|x, p=-\bar{p}=0\rangle .
$$

\footnotetext{
${ }^{4}$ The idea used in $[10]$ where the path integral representation was used to support open string Tduality has been generalized. Here as the result of a T-duality in an orthogonal direction the boundary state of $D_{p}$ brane can be constructed from the boundary state of $D_{-1}$ brane. In 2.24 ) a further Tduality in a direction tanget to a Dp-brane is equivalent to changing the measure $\prod_{i=0}^{p} D x_{i}(\sigma)$ to $\prod_{i=0}^{p-1} D x_{i}(\sigma)$.
} 
Firstly this above solution ${ }^{5}$ can be integrated out to show the final result is right the boundary state in the constant $U(1)$ gauge field background in [2]. The final result of (2.28) in the Euclidean spacetime is

$$
|B, p=0\rangle=N(F) \exp \left(-\sum_{n=1}^{\infty} \frac{1}{n} \alpha_{-n}\left(\frac{1-F}{1+F}\right) \tilde{\alpha}_{-n}\right)|p=-\bar{p}=0\rangle
$$

with the normalization factor $N(F)=(\operatorname{det}(1+F))^{\frac{1}{2}}$. In bosonic string case the normalization factor is just the effective action of gauge potential in [11] ${ }^{6}$.

The purpose of arguing the path integral representation in 90 is to obtain the normalization factor. Here shows this approach to get normalization factor is rather natural in contrast with the other methods. The method in [2] is to compare the one point amplitude of the boundary state with that from Dirac-Born-Infeld action. In addition the detailed form of the normalization factor in [8] is in the requirement of gauge invariance of D-brane source term contained in the action of closed string field theory.

With the form (2.19) and the following

$$
\partial_{\sigma} X(\sigma)=-\sum_{n \neq 0}\left(\alpha_{n}-\tilde{\alpha}_{-n}\right) e^{i n \sigma}
$$

the path integral representation of (2.28) may be written with another form

$$
|B, p=0\rangle=\int[d x] \exp \left(\frac{i}{8 \pi} F_{\mu \nu} \int_{0}^{2 \pi} d \sigma x^{\mu}(\sigma) \cdot \partial_{\sigma} x^{\nu}\right)|x, p=-\bar{p}=0\rangle
$$

which shows the boundary interaction of $U(1)$ constant gauge field background.

With the help of (2.23), the above boundary state can be changed into the form

$$
|B, p=0\rangle=\int D x(\sigma) \exp \left(\frac{i}{8 \pi} F_{\mu \nu} \int_{0}^{2 \pi} d \sigma x^{\mu}(\sigma) \cdot \partial_{\sigma} x^{\nu}-i \int_{0}^{2 \pi} d \sigma P_{\mu} \cdot x^{\mu}\right)|B\rangle_{-1}
$$

\footnotetext{
${ }^{5}$ It is nontrivial to point out that in the presence of a constant $U(1)$ gauge field background Virasoro generators are not modified.

${ }^{6}$ We would like to thank Professor A.A.Tseytlin for bringing our attention to the paper [11]. In fact, our purpose which is different from of [1] is to give an interesting example to show potential value of path integral representation. The familiar boundary state in $U(1)$ constant gauge background can be only determined by conformal invariance. The boundary state can be used to determine boundary condition from which the action can be constructed. Naturally this example also shows the effective action or the normalization factor can be determined directly from the conformal invariance.
} 
from which the boundary condition determining such above boundary state may be found. Indeed, one can show that the following identity holds:

$$
\begin{aligned}
0 & =\int D x(\sigma) \frac{\delta}{\delta x^{\mu}(\sigma)} \exp \left(\frac{i}{8 \pi} F_{\mu \nu} \int_{0}^{2 \pi} d \sigma x^{\mu}(\sigma) \cdot \partial_{\sigma} x^{\nu}-i \int_{0}^{2 \pi} d \sigma P_{\mu} \cdot x^{\mu}\right)|B\rangle_{-1} \\
& =\left[\frac{i}{4 \pi} F_{\mu \nu} \partial_{\sigma} X^{\nu}-i P_{\mu}(\sigma)\right]|B, p=0\rangle
\end{aligned}
$$

where the boundary condition

$$
\left(\partial_{\tau} X_{\mu}-F_{\mu \nu} \partial_{\sigma} X^{\nu}\right)|B, p=0\rangle=0
$$

can be extracted. Here it is both the boundary condition and the conformal invariance which require

$$
p=-\bar{p}=0 .
$$

Such the boundary condition just corresponds to the open string theory

$$
S=\int d \tau \int_{0}^{2 \pi} d \sigma\left\{\frac{1}{4 \pi \alpha^{\prime}}\left[\left(\partial_{\tau} X\right)^{2}-\left(\partial_{\sigma} X\right)^{2}\right]-[\delta(\sigma)-\delta(\sigma-2 \pi)] \dot{X}^{\mu} A_{\mu}(X)\right\}
$$

The following is a simple review to [6] where the path integral representation of the boundary state in the $U(1)$ constant gauge field background can be also argued from the point of Matrix theory. Notice that their conventions are a little different with ours since the definition of $X(\tau, \sigma)$ and two order antisymmetry tensor $F_{\mu \nu}$ are not completely fixed.

The configuration of infinitely many D-instantons can be expressed by $\infty \times \infty$ hermitian matrices $X^{M}(M=0, \cdots, D-1)$. The one we consider is

$$
\begin{aligned}
X^{i} & =\hat{Q}^{i},(i=0, \cdots, p) \\
X^{M} & =0(M=p+1, \cdots, D-1),
\end{aligned}
$$

where $\hat{Q}^{i}(i=0 \cdots, p)$ satisfy

$$
\left[\hat{Q}^{i}, \hat{Q}^{j}\right]=i \theta^{i j}
$$

Here $(p+1) \times(p+1)$ matrix $\theta$ is assumed to be invertible.

In Matrix theory this configuration of D-instantons is equivalent to a $\mathrm{D} p$-brane. $\mathrm{A}$ quick way to see the equivalence is to look at the boundary states. The boundary state $|B\rangle$ corresponding to the configuration eq. (2.37) can be written as follows:

$$
|B\rangle=\operatorname{Tr} X e^{-i \int_{0}^{2 \pi} d \sigma P_{i} \cdot \hat{X}^{i}}|B\rangle_{-1}
$$


$|B\rangle_{-1}$ includes also ghost part which is not relevant to the discussion here. The factor in front of $|B\rangle_{-1}$ is an analogue of Wilson loop and corresponds to the background eq. (2.37). Eq. (2.39) can be rewritten with the path integral as

$$
|B, p=0\rangle=\int D x(\sigma) \exp \left(\frac{i}{2} \int d \sigma x^{i} \cdot \partial_{\sigma} x^{j} F_{i j}-i \int d \sigma P_{i} \cdot x^{i}\right)|B\rangle_{-1}
$$

where $F_{i j}=\left(\theta^{-1}\right)_{i j}$.

It is straightforward to perform the path integral in eq. (2.40). In conclusion $|B\rangle$ is equivalent to the boundary state for a $\mathrm{D} p$-brane with $U(1)$ gauge field strength $F_{i j}$ on the worldvolume.

\subsection{Some notes of the path integral representation of the boundary state}

To construct a good theory of $D_{p}$ brane in the general background, we can argue

$$
|B\rangle_{p}^{N}=\int \prod_{i=0}^{p} D x_{i}(\sigma) \exp \left(S\left(\hat{q}, x_{n}, \frac{\partial}{\partial x_{n}}, \cdots\right)\right)|x(\sigma)\rangle,
$$

where $|B\rangle_{p}^{N}$ is the Neumann part of the boundary state $|B\rangle_{p}$ which is defined as

$$
|B\rangle_{p}=|B\rangle_{p}^{N} \cdot|B\rangle_{p}^{D}
$$

with $|B\rangle_{p}^{D}=\prod_{\mu=p+1}^{D-1}|B\rangle_{-1}^{\mu}$. In essence 2.41) may be seen as Fourier transform between the boundary state $|B\rangle_{p}^{N}$ with boundary interaction and the coherent state $|x(\sigma)\rangle^{7}$.

For the path integral representation of the boundary state

$$
|B, p\rangle=\int[d x]|x, p=-\bar{p}\rangle \exp (S(x))
$$

we can check those equations

$$
\begin{aligned}
\left(\sum_{m \neq 0}^{\infty} m x_{m+n} \frac{\partial}{\partial x_{m}}\right) S(x) & =0, n \neq 0, \\
\sum_{m \neq 0}^{\infty} m x_{m} \frac{\partial}{\partial x_{m}} S(x) & =0, \\
x_{0} & =0
\end{aligned}
$$

\footnotetext{
${ }^{7}$ From the above expression, it can be argued that physics of the world volume of $D_{p}$ brane, which is represented by $|B\rangle_{p}^{N}$, may be equivalent to the complete physics of $(p+1)$ particles in space time, which is represented by quantum state $\prod_{i=0}^{p}|x(\sigma)\rangle^{i}$. The involved motion of these quantum states is with the weight $\left(S\left(\hat{q}, x_{n}, \frac{\partial}{\partial x_{n}}, \cdots\right)\right)$ which is determined by the respective paths.
} 
from the conformal invariance are consistent with the following equations

$$
\begin{aligned}
2 n \frac{\partial}{\partial x_{n}^{\mu}} S(x) & =F_{\mu \nu} x_{-n}^{\nu}, n \neq 0, \\
P & =0
\end{aligned}
$$

from the mixed boundary condition determining the boundary state in the constant $U(1)$ gauge field background. Therefore when we take the ansatz $S(x)$, we will know such path integral representation shows the physics of $D$ brane in constant $U(1)$ gauge field background before the detailed path integral calculation in Appendix A. In fact it is important to firstly estimate the form of $S$ with some physical consideration before determining the detailed form of $S$ by the conformal invariance.

In this paper some ansatz can be changed. Define the coherent state

$$
\left(\alpha_{n}+\tilde{\alpha}_{-n}-p_{n}\right)|p p\rangle=0
$$

then the boundary state with the boundary interaction can be expanded into the sum of the above coherent state,

$$
|B, P\rangle=\int[d p] \exp \left(S\left(\hat{q}, p_{n}, \frac{\partial}{\partial p_{n}}, \cdots\right)\right)|p p\rangle=0 .
$$

So all the calculation in this paper can be carried out from the new starting point.

\section{The path integral representation of the boundary state in other special backgrounds}

Besides the $U(1)$ gauge field background, the linear dilaton background and the tachyon background are important in recent research. Especially, it seems attracting how to construct the boundary state of D-brane in Type 0 string under the closed string tachyon background and make it consistent with the argument that D-brane is the classical soliton solution with R-R charge.

\subsection{The path integral representation of the boundary state in the linear dilaton background}

We will construct the conformal invariant boundary state in the linear dilaton background with path integral representation ${ }^{8}$.

\footnotetext{
${ }^{8}$ Professor Miao Li introduced me his paper [12] and advised me to read this paper carefully. In [12] the conformal invariance was directly used to construct the path integral representation of boundary state in the linear dilaton background. However the path integral representation of the boundary state in 12 can be found not to be exactly conformal invariant. In the following the conformal invariant boundary state will be constructed.
} 
Now the starting point is still the stress tensor

$$
T=-\frac{1}{2}(\partial \phi)^{2}+Q \partial^{2} \phi
$$

and a similar anti-holomorphic counterpart. The central charge of this free scalar is $c=1+12 Q^{2}$, and $Q=\sqrt{2}$ in two dimensional string theory. Consider a unit disk, the conformal invariance condition on the boundary means no net energy-momentum flow out of the boundary. It is convenient to work with mode expansions

$$
\begin{aligned}
\phi & =\varphi_{0}-i(p \ln z+\bar{p} \ln \bar{z})-i \sum_{n \neq 0} \frac{1}{n}\left(\alpha_{-n} z^{n}+\tilde{\alpha}_{-n} \bar{z}^{n}\right), \\
L_{n} & =\left[\alpha_{0}+i Q(n+1)\right] \alpha_{n}+\frac{1}{2} \sum_{m \neq 0,-n} \alpha_{m+n} \alpha_{-m}, \quad n \neq 0, \\
L_{0} & =\left[\frac{\alpha_{0}}{2}+i Q\right] \alpha_{0}+\frac{1}{2} \sum_{m \neq 0} \alpha_{m} \alpha_{-m} .
\end{aligned}
$$

The similar formula for $\tilde{L}_{n}$. The commutators are $\left[\alpha_{m}, \alpha_{n}\right]=m \delta_{m+n, 0}$, and similarly for the right-moving modes. Let $K_{n}=L_{n}-\tilde{L}_{-n}$. The boundary condition is entirely encoded in the boundary state $|B\rangle$, and the conformal invariance condition is $K_{n}|B\rangle=$ 0 .

The usual Neumann boundary condition is given by $\partial_{r} \phi=0$ on the boundary of the unit disk. In terms of the boundary state, it states that

$$
P|B\rangle_{N}=\left(\alpha_{n}+\tilde{\alpha}_{-n}\right)|B\rangle_{N}=0
$$

Due to the existence of the background charge $Q$, one has to modify the boundary condition a bit: $p=-i Q$. So there must be a net momentum flow out of the boundary (in view of spacetime $\phi$ ). One way to see this is to consider the commutators

$$
\left[K_{m}, \alpha_{n}+\tilde{\alpha}_{-n}\right]=2 i Q m \delta_{m+n, 0}-n\left(\alpha_{m+n}+\tilde{\alpha}_{-m-n}\right)
$$

When the case $n+m=0$ occurs, the above commutator can be showed in the clearer form

$$
\left[K_{m}, \alpha_{n}+\tilde{\alpha}_{-n}\right]=m\left(2 i Q+\alpha_{0}+\tilde{\alpha}_{0}\right)
$$

Actually, we have taken the following conventions,

$$
2 p=\alpha_{0}+\tilde{\alpha}_{0}, \quad \alpha_{0}=\tilde{\alpha}_{0}=p .
$$

So when $p=-i Q$, the center term disappears, and it is possible to impose both the conformal invariance condition and Neumann boundary condition. To be as close to 
the ordinary Dirichlet condition as possible, one requires that a net momentum transfer is possible if one scatters string states against the object described by the boundary state. So $|B, p\rangle$ is an eigen-state of $p$ with arbitrary number $p$. To solve equations $K_{n}|B, p\rangle=0$, we may construct the path integral representation of the conformal invariant boundary state. However the ansatz $S(x, \hat{q}, \hat{\tilde{q}})$ is one little special form with the contribution of zero mode,

$$
S(x, \hat{q}, \hat{\tilde{q}})=V_{\text {coup }}^{n} \exp \left(\frac{\hat{q}+\hat{\tilde{q}}}{2 Q}\right) \Phi(x),
$$

where the operator $\hat{q}$ and $\hat{\tilde{q}}$ satisfy the commutative relation $[\hat{q}, \hat{\tilde{q}}]=0,\left[\alpha_{0}, \hat{q}\right]=-i$ and $\left[\tilde{\alpha}_{0}, \hat{\tilde{q}}\right]=-i$. And $V_{\text {coup }}^{n}$ is the coupling constant before renormalization procedure. The boundary state has been changed into the form

$$
|B\rangle=\int[d x] \exp \left(V_{\text {coup }}^{n} \exp \left(\frac{\hat{q}+\hat{\tilde{q}}}{2 Q}\right) \Phi(x)\right)|x, p=\bar{p}=-i Q\rangle .
$$

So the equation $K_{n}|B, p\rangle=0$ now can be changed into the following two equations,

$$
\begin{aligned}
\frac{-i}{2 Q} x_{n} \Phi(x)+2 i Q n^{2} \frac{\partial}{\partial x_{-n}} \Phi(x) & =\sum_{m \neq 0,-n}^{\infty} m x_{m+n} \frac{\partial}{\partial x_{m}} \Phi(x), \\
\sum_{m \neq 0}^{\infty} m x_{m} \frac{\partial}{\partial x_{m}} \Phi(x) & =0 .
\end{aligned}
$$

The special solution of the two equations could be found as follow,

$$
\Phi(x)=\oint \frac{d z}{z} \exp \left(\sum_{m \neq 0} \frac{i x_{m}}{2 Q m z^{m}}\right) .
$$

As we have claimed, such above solution contains the divergence. Naturally the renormalized boundary state which will be given in Appendix B is conformal invariant only in some specific cases.

Actually the conformal invariant boundary state in the linear dilaton background can be constructed without considering the path integral representation. Define the screening charge

$$
Q Q=\oint d z: \exp (i k \cdot X(z)):
$$

with $k$ must satisfy the following equation

$$
k^{2}+2 i Q \cdot k=2 .
$$


Since the commutative relation $\left[L_{n}, Q Q\right]=0$, the conformal invariant boundary state 9 is

$$
|B\rangle=F u n(Q Q)|B, p=\bar{p}=-i Q\rangle_{N}
$$

in which Fun is function of $Q Q$. The boundary state $|B, p=\bar{p}=-i Q\rangle_{N}$ is the Neumanm boundary state with the momemtum which satisfies

$$
\begin{aligned}
\left(\alpha_{n}+\tilde{\alpha}_{-n}\right) \mid B, p & =\bar{p}=-i Q\rangle_{N}=0, n \neq 0, \\
\left(\alpha_{0}+\tilde{\alpha}_{0}\right)|B, p=\bar{p}=-i Q\rangle_{N} & =2 p|B, p=\bar{p}=-i Q\rangle_{N}, \\
\left(L_{n}-\tilde{L}_{-n}\right) \mid B, p & =\bar{p}=-i Q\rangle_{N}=0 .
\end{aligned}
$$

Here the boundary state (3.13)is still rather specifically. In principle the number of conformal invariant boundary state in the linear dilaton background is infinitely many.

\subsection{The path integral representation of the boundary state in the open string tachyon background}

The path integral representation of the boundary state in the open string tachyon background will be constructed, which provides some clues to construct the conformal invariant boundary state of D-brane from Type 0 string in the tachyon background ${ }^{10}$.

The conformal invariant boundary state in the open string tachyon background 11 is the form

$$
|B\rangle=\exp (\oint d z: \exp (i k \cdot X(z)):)|B, p=-\bar{p}\rangle_{N},
$$

with the convention $k^{2}=2$. So the path integral representation of the boundary state in the open string tachyon background will have one form

$$
\begin{array}{r}
|B\rangle=\int[d x]|x, p=-\bar{p}\rangle+ \\
\int[d x] \sum_{l=1}^{\infty} \Phi_{l}|x, p=-\bar{p}\rangle .
\end{array}
$$

\footnotetext{
${ }^{9}$ This conformal invariant boundary state is useful in Appendix B.

${ }^{10} \mathrm{It}$ is necessary to point out that tachyon appearing in Type 0 string theory is closed string state but here tachyon is open string state. Since the boundary state is the closed string source, it is possible to construct the boundary state of D-brane in Type 0 string. On the other hand in order to support the argument the path integral representation of boundary state can provide an exact description of D-brane in general background, we have to give a nontrivial example. So the work to ensure the boundary state representing the boundary state of D-brane in Type 0 string is just nontrivial.

${ }^{11}$ The boundary state in the open string tachyon background has been researched in [13].
} 
The differential equations from the conformal invariance constraint are

$$
\Phi_{l}\left(-n l \frac{\partial}{\partial x_{-n}}+\frac{1}{2} l x_{n}\right)+\Phi_{l}\left(\sum_{m \neq 0} m x_{m+n} \frac{\partial}{\partial x_{m}}\right)=0, \quad n \neq 0,
$$

and for $n=0$ case the differential equation is

$$
\Phi_{l}\left(\frac{1}{2} l^{2} k^{2}+l k \cdot p\right)+\Phi_{l}\left(\sum_{m \neq 0} m x_{m} \frac{\partial}{\partial x_{m}}\right)=0 .
$$

Finally the ansatz of $\Phi_{l}$, namely $\Phi_{l}\left(\hat{q}, p, x_{n}, x_{-n}, \frac{\partial}{\partial x_{n}}\right)$ is solved as

$$
\begin{aligned}
& \Phi_{l}=\prod_{i=1}^{l} \oint d z_{i} \exp ^{i k \cdot \hat{q}} z_{i}^{k \cdot p} z_{i}^{(i-1) k^{2}} \\
& \exp \left(\sum_{n=1} \frac{k \cdot x_{n}}{-2 n z_{i}^{n}}\right) \exp \left(\sum_{n \neq 0} k z_{i}^{n} \cdot \frac{\partial}{\partial x_{n}}\right) \exp \left(\sum_{n=1} \frac{k \cdot x_{-n} z_{i}^{n}}{2 n}\right),
\end{aligned}
$$

with the definition of $\prod_{i=1}^{l} \oint\left[d z_{i}\right] \equiv \oint\left[d z_{1}\right] \oint\left[d z_{2}\right] \cdots \oint\left[d z_{l}\right]$. In essence this solution is relevant with the form of (3.15). The factor $z_{i}^{(i-1) k^{2}}$ appearing in the (3.20) is from the noncommutative relation between zero modes, for example $l=2$ case,

$$
\begin{aligned}
& \oint d z_{2} \exp ^{i k \cdot \hat{q}} z_{2}^{k \cdot \hat{p}} \oint d z_{1} \exp ^{i k \cdot \hat{q}} z_{1}^{k \cdot p}|x, p=-\bar{p}\rangle \\
& =\oint d z_{1} \exp ^{i k \cdot \hat{q}} z_{1}^{k \cdot p} \oint d z_{2} \exp ^{i k \cdot \hat{q}} z_{2}^{k \cdot p} z_{2}{ }^{2}|x, p=-\bar{p}\rangle .
\end{aligned}
$$

Although such above calculation is not easy, it shows the path integral representation of the boundary state is very useful if we can take some tricks in the given background.

\section{Discussions}

In this paper some examples of the path integral representation of the boundary state are given in some special backgrounds such as the $U(1)$ gauge field background, the linear dilaton background and the open string tachyon background. The path integral representation of the boundary state in the $U(1)$ gauge field background contains much essential information, especially the normalization factor which is the effective action of gauge field in the bosonic string theory. The application in Matrix theory and Noncommutative geometry hints that Path integral representation of the boundary state could be rather fundamental in describing D-brane in the general background. 
The initial purpose of this paper is to construct a general solution of the boundary conformal field theory with analytical approach, mainly for the constraint equations $\left(L_{n}-\tilde{L}_{-n}\right)|B\rangle=0$ are difficult to be solved to obtain the solution represented by string modes from the pure algebraic approach. However in the path integral representation it is easy transforming those algebraic equations into the differential formalism which can be solved. In addition, Cardy condition which ensures the existence of open string theory in the boundary conformal field theory is vital to this view point that the boundary conformal field theory is an exact description of D-brane in the general background. We will consider Cardy condition realization in the path integral representation in the future work. Finally, it is also our wish that the path integral representation of boundary state should be used to support or interpret the known ansatz in [3] ${ }^{12}$,

$$
\left\langle\exp \int_{S^{d}} \phi_{0} O\right\rangle_{C F T}=Z_{S}\left(\phi_{0}\right) .
$$

It is possible to supersymmetrize all the results in this paper. There are still a lot of work to be done. The path integral representation of constant gauge field configuration should be generalized to nonconstant background in the conformal invariant requirement. The path integral representation of the boundary state in the linear dilaton background might be useful to construct the boundary state under $A d S_{3}$ background, especially in the light cone gauge in [17]. Most important, the construction of the boundary state in tachyon background is potentially useful for the generalization of $A d S / C F T$ in non-super symmetrical case. The recent research on D-brane in Type 0 string shows we must face the tachyon problem in arriving at the purpose. However, it seems that the usual boundary state is not reasonable which is constructed from the general procedure without considering the tachyon background effect, because the classical p-brane from the usual boundary state [18] is not the solution in [19]. This can be the result of the introduction of the tachyon background, which makes the equations corresponding to p-brane solution become nonlinear. But the usual boundary state is only the linear combination of closed string states. Since nonsupersymmetrical generalization of $A d S / C F T$ in Type 0 string case seems more reasonable than in that

\footnotetext{
${ }^{12}$ It has been recently argued that holographic principle should be deduced from noncommutative geometry, such as in 14. And it seems that two fundamental principles are intrinsic consistent in [15. In fact they can be both argued from the point of string/M theory. So it is possible to deduce AdS/CFT duality from string/M theory. Naturally we wish that this procedure may be depend on the path integral representation of boundary state. From string amplitude with D brane, which may be represented by saturating string state with boundary state in[2], much useful information about supergravity can be obtained in 16 . However those cases are in flat spacetime, but now we have to face curved spacetime such as AdS. We wish we could continue this research because this will provide a truely nontrivial example of path integral representation of boundary state of D-brane.
} 
approach of supersymmetrical breaking case used in [20], it is possible to construct the boundary state with the tachyon background in Type 0 string case.

\section{Acknowledgments}

We would like to thank Miao Li for helpful comments on the manuscript and Yi-Hong Gao for a helpful discussion.

\section{A. The calculation of the normalization factor $N(F)$}

In the following the calculation of the normalization factor $N(F)$ is given. The special solution of the differential equation (2.13) and the differential equation (2.14) is

$$
\begin{aligned}
S(x) & =\frac{1}{4} F_{\mu \nu} \sum_{m \neq 0} \frac{x_{m}{ }^{\mu} x_{-m}{ }^{\nu}}{m}, \\
& =\frac{-1}{2} F_{\mu \nu} \sum_{m=1}^{\infty} \frac{x_{-m}{ }^{\mu} x_{m}{ }^{\nu}}{m} .
\end{aligned}
$$

The entire calculation of the path integral of (2.28) has to be put in the Euclidean spacetime. The calculation of the (2.28) is as follow,

$$
\begin{aligned}
|B, p=0\rangle= & \int[d x] \exp \left(\frac{1}{4} F_{\mu \nu} \sum_{m \neq 0} \frac{x_{m}{ }^{\mu} x_{-m}{ }^{\nu}}{m}\right)|x, p=-\bar{p}=0\rangle, \\
= & \int[d x] \exp \left(\frac{-1}{2} \sum_{m=1}^{\infty} \frac{1}{m}\left(x_{-m}-2 \alpha_{-m} \frac{1}{1+F}\right)(1+F)\left(x_{m}+2 \frac{1}{1+F} \tilde{\alpha}_{-m}\right)\right) \\
& \exp \left(-\sum_{m=1}^{\infty} \frac{1}{m} \alpha_{-m}\left(\frac{1-F}{1+F}\right) \tilde{\alpha}_{-m}\right)|p=-\bar{p}=0\rangle .
\end{aligned}
$$

The integral measure in the above formula has to be chosen as

$$
\begin{aligned}
\int[d x] & \equiv \int \prod_{n=1}^{\infty} \frac{d x_{-n} d x_{n}}{n}, \\
\int \frac{d x_{-n} d x_{n}}{n} & \equiv \int \frac{d a d b}{(2 \pi)^{D}}
\end{aligned}
$$

with the definition of $x_{n}=\sqrt{n}(a+i b)$ and $x_{-n}=\sqrt{n}(a-i b)$. The choice of (A.4) aims at using the following formula in our calculation,

$$
\int \frac{d a d b}{(2 \pi)^{D}} \exp \left(\frac{-1}{2}(a-i b) A(a+i b)\right)=\frac{1}{A^{D}} .
$$


So the final result can be given,

$$
\begin{aligned}
|B, p=0\rangle= & \int[d x] \exp \left(\frac{1}{4} F_{\mu \nu} \sum_{m \neq 0} \frac{x_{m}{ }^{\mu} x_{-m}{ }^{\nu}}{m}\right)|x, p=-\bar{p}=0\rangle, \\
= & (\operatorname{Det}(1+F))^{-1} \exp \left(-\sum_{m=1}^{\infty} \frac{1}{m} \alpha_{-m}\left(\frac{1-F}{1+F}\right) \tilde{\alpha}_{-m}\right) \\
& |p=-\bar{p}=0\rangle .
\end{aligned}
$$

In conclusion, the normalization factor should take the form

$$
N(F)=(\operatorname{Det}(1+F))^{-1} \text {. }
$$

Since

$$
\begin{aligned}
& \operatorname{Det}(1+F)=\prod_{m=1}^{\infty} \operatorname{det}(1+F), \\
& \sum_{m=1}^{\infty} 1=\zeta(0)=\frac{-1}{2},
\end{aligned}
$$

we obtain

$$
N(F)=(\operatorname{det}(1+F))^{\frac{1}{2}}
$$

\section{B. The conformal path integral representation of the boundary state in the linear dilaton background}

The path integral representation of the boundary state in the linear dilaton background is the form,

$$
|B\rangle=\int[d x] \exp \left(V_{\text {coup }}^{n} \exp \left(\frac{\hat{q}+\hat{\tilde{q}}}{2 Q}\right) \Phi(x)\right)|x, p=\bar{p}=-i Q\rangle .
$$

The conformal invariant constraint of the boundary state, $\left(L_{n}-\tilde{L}_{-n}\right)|B\rangle=0$, can be expressed by

$$
\begin{aligned}
\left(L_{n}-\tilde{L}_{-n}\right)|B\rangle= & \int[d x]\left[\alpha_{0} \alpha_{n}-\tilde{\alpha}_{0} \tilde{\alpha}_{-n}, \exp S(x, \hat{q}, \hat{\tilde{q}})\right]|x, p=\bar{p}=-i Q\rangle+ \\
& \int[d x] \exp S(x, \hat{q}, \hat{\tilde{q}})\left(-2 i Q n^{2} \frac{\partial}{\partial x_{-n}}+\sum_{m \neq 0,-n}^{\infty} m x_{m+n} \frac{\partial}{\partial x_{m}}\right) \\
& |x, p=\bar{p}=-i Q\rangle=0 .
\end{aligned}
$$


So the equation $K_{n}|B, p\rangle=0$ now can be changed into the following two equations,

$$
\begin{aligned}
2 i Q n^{2} \frac{\partial}{\partial x_{-n}} \Phi(x) & =\frac{i}{2 Q} x_{n} \Phi(x)+\sum_{m \neq 0,-n}^{\infty} m x_{m+n} \frac{\partial}{\partial x_{m}} \Phi(x), \quad n \neq 0, \\
\sum_{m \neq 0}^{\infty} m x_{m} \frac{\partial}{\partial x_{m}} \Phi(x) & =0
\end{aligned}
$$

with the commutative relation

$$
\begin{aligned}
& {\left[\alpha_{0} \alpha_{n}-\tilde{\alpha}_{0} \tilde{\alpha}_{-n}, \exp S(x, \hat{q}, \hat{\tilde{q}})\right]} \\
& =\frac{-i}{2 Q}\left(\alpha_{n}-\tilde{\alpha}_{-n}\right) S(x, \hat{q}, \hat{\tilde{q}}) \exp S(x, \hat{q}, \hat{\tilde{q}}) .
\end{aligned}
$$

The special solution of the two equations could be found,

$$
\Phi(x)=\oint \frac{d z}{z} \exp \left(\sum_{m \neq 0} \frac{i x_{m}}{2 Q m z^{m}}\right) .
$$

Now we come to check the solution. The right part of the (B.4) is

$$
\begin{aligned}
& \frac{i}{2 Q} x_{n} \Phi(x)+\sum_{m \neq 0,-n}^{\infty} m x_{m+n} \frac{\partial}{\partial x_{m}} \Phi(x) \\
& =\oint d z\left(-z^{n}\right) \frac{d}{d z} \exp \left(\sum_{m \neq 0} \frac{i x_{m}}{2 Q m z^{m}}\right) ;
\end{aligned}
$$

and the left part of the $(\overline{\mathrm{B} .4})$ is

$$
2 i Q n^{2} \frac{\partial}{\partial x_{-n}} \Phi(x)=\oint d z n z^{n-1} \exp \left(\sum_{m \neq 0} \frac{i x_{m}}{2 Q m z^{m}}\right) ;
$$

so the difference between the above expressions is zero integral ${ }^{13}$. With $\left(\alpha_{n}-\tilde{\alpha}_{-n}-\right.$ $\left.x_{n}\right)|x, p=\bar{p}=-i Q\rangle=0$, and $\hat{q}|x, p=\bar{p}=-i Q\rangle=\hat{\tilde{q}}|x, p=\bar{p}=-i Q\rangle$, the boundary state is transformed into

$$
\begin{aligned}
|B\rangle & =\int[d x] \exp \left(V_{\text {coup }}^{n} \exp \left(\frac{\hat{q}+\hat{\tilde{q}}}{2 Q}\right) \Phi(x)\right)|x, p=\bar{p}=-i Q\rangle \\
& =\exp \left(V_{\text {coup }}^{n} \exp \left(\frac{\hat{q}+\hat{\tilde{q}}}{2 Q}\right) \oint \frac{d z}{z} \exp \left(\sum_{m \neq 0} \frac{i\left(\alpha_{m}-\tilde{\alpha}_{-m}\right)}{2 Q m z^{m}}\right)\right)|B, p=\bar{p}=-i Q\rangle_{N} \\
& =\exp \left(V_{\text {coup }}^{n} \exp \left(\frac{\hat{q}}{Q}\right) \oint \frac{d z}{z} \exp \left(\sum_{m \neq 0} \frac{i \alpha_{m}}{Q m z^{m}}\right)\right)|B, p=\bar{p}=-i Q\rangle_{N}
\end{aligned}
$$

\footnotetext{
${ }^{13}$ if $x_{0} \neq 0$ then the conclusion of the zero integral is not correct.
} 
which contains the divergence, so needs normalizing and renormalization. With the commutator $\left[\alpha_{n}, \alpha_{m}\right]=n(1-\epsilon)^{|n|} \delta_{n+m, 0}$, the expression $\exp \left(\sum_{m \neq 0} \frac{i \alpha_{m}}{Q m z^{m}}\right)$ may be represented by

$$
\exp \left(\sum_{m=1}^{\infty} \frac{i \alpha_{-m} z^{m}}{-Q m}\right) \exp \left(\sum_{m=1}^{\infty} \frac{i \alpha_{m}}{Q m z^{m}}\right)\left(\epsilon^{\frac{-1}{2} Q^{2}}\right)
$$

With the renormalized coupling constant $V_{\text {coup }}^{r}$, the boundary state in the path integral representation has the final form

$$
|B\rangle=\exp \left(V_{\text {coup }}^{r} \exp \left(\frac{\hat{q}}{Q}\right) \oint \frac{d z}{z} \exp \left(\sum_{m=1}^{\infty} \frac{\alpha_{-m} z^{m}}{i Q m}\right) \exp \left(\sum_{m=1}^{\infty} \frac{\alpha_{m}}{-i Q m z^{m}}\right)\right)|B, p=\bar{p}=-i Q\rangle_{N} .
$$

Since the boundary state $|B, p=\bar{p}=-i Q\rangle_{N}$ is conformal invariant, we have to verify that the former part of $|B, p=\bar{p}=-i Q\rangle_{N}$ should commute with Virasoro generator $L_{n}$ to ensure the conformal invariance of $|B\rangle$. In fact that part is similar to the screening charge (3.11),

$$
\begin{aligned}
Q Q= & \oint d z: \exp (i k \cdot X(z)): \\
= & \oint d z \exp (i k \cdot \hat{q}) z^{k \cdot \hat{p}} \exp \left(\sum_{m=1}^{\infty} \frac{k \cdot \alpha_{-m} z^{m}}{m}\right) \exp \left(\sum_{m=1}^{\infty} \frac{k \cdot \alpha_{m}}{-m z^{m}}\right) \\
& |B, p=\bar{p}=-i Q\rangle_{N},
\end{aligned}
$$

with $k$ must satisfy the following equation,

$$
k^{2}+2 i Q \cdot k=2 .
$$

With $k=\frac{1}{i Q}$, the screening charge is just the former factor of the first order term of coupling constant of the boundary state. In addition, the solutions of equation (B.13) may be labeled by $\frac{1}{a_{+}}$or $\frac{1}{a_{-}}$. The $a_{+}$and $a_{-}$are respectively $\frac{1}{2}\left(Q+\sqrt{Q^{2}-2}\right)$ and $\frac{1}{2}\left(Q-\sqrt{Q^{2}-2}\right)$. So the solution $k=1 /(i Q)$ is good in the large $\mathrm{Q}$ limit with the corrections suppressed by $1 / Q^{2}$ etc. to $k$. Such a limit will change the boundary state $|B\rangle$ into $|B, p=\bar{p}=-i Q\rangle_{N}$ which is conformal invariant. Therefore it is reasonable that the renormalized boundary state should be conformal invariant in some special backgrounds.

\section{References}

[1] J. Dai, R. G. Leigh and J. Polchinski, Mod. Phys. Lett. A4 (1989) 2073;

J. Polchinski, hep-th/9510017, Phys. Rev. Lett. 75 (1995) 4724. 
[2] Matthias R Gaberdiel, hep-th/0005029;

Ben Craps, hep-th/0004198;

Paolo Di Vecchia, Antonella Liccardo, hep-th/9912161, hep-th/9912275.

[3] Edward Witten, hep-th/9802150, Adv. Theor. Math. Phys. 2 (1998) 505-532.

[4] John L. Cardy, Nucl. Phys. B324 (1989) 581.

N. Ishibashi, Mod. Phys. Lett. A4 (1989) 251;

N. Ishibashi and T. Onagi, Mod. Phys. Lett. A4 (1989) 161.

[5] A. Recknagel, V. Schomerus, hep-th/9712186, Nucl. Phys. B531 (1998) 185-225.

[6] Nobuyuki Ishibashi, hep-th/9804163, Nucl. Phys. B539 (1999) 107-120.

[7] N. Ishibashi, H. Kawai, Y. Kitazawa, A. Tsuchiya, hep-th/9908141, Nucl. Phys. B565 (2000) 176-192;

N. Ishibashi, hep-th/9909176;

Kazumi Okuyama, hep-th/9910138, JHEP 0003 (2000) 016;

Mitsuhiro Kato, Tsunehide Kuroki, hep-th/9902004, JHEP 9903:012, 1999.

[8] Koji Hashimoto, Hiroyuki Hata, hep-th/9704125, Phys. Rev. D56 (1997) 5179-5193;

Koji Hashimoto, hep-th/9909027, Phys. Rev. D61 (2000) 106002;

Koji Hashimoto, hep-th/9909095.

[9] C. G. Callan, C. Lovelace, C. R. Nappi and S. A. Yost, Nucl.Phys.B 293 (1987) 83; Nucl. Phys. B 308 (1988) 221.

[10] E. Alvarez, J. L. F. Barbon, J. Borlaf, hep-th/9603089, Nucl. Phys. B479 (1996) 218-242.

[11] E.S.Fradkin and A.A.Tseytlin, Phys.Lett.163B (1985) 123;

A.Abouelsaood, C.G.Callan, C.R.Nappi, and S.A.Yost, Nucl. Phys.B280 (1987) 599-624;

A. A. Tseytlin, hep-th/9908105.

[12] Miao. Li, hep-th/9512042, Phys. Rev. D54 (1996) 1644-1646.

[13] Ali Yegulalp, hep-th/9504104, Nucl. Phys. B450 (1995) 641;

G. Callan, Igor R. Klebanov, Andreas W. W. Ludwig and Juan M. Maldacena, hepth/9402113, Nucl. Phys. B422 (1994) 417-448.

[14] Zhe Chang, hep-th/9904101, Phys.Rev. D61 (2000) 044009.

[15] Miao Li, Yong-Shi Wu, Phys.Rev.Lett. 84 (2000) 2084-2087, hep-th/9909085.

[16] I.R. Klebanov and L. Thorlacius, Phys. Lett. B371 (1996) 51 hep-th/9510200;

S.S. Gubser, A. Hashimoto, I.R. Klebanov and J.M. Maldacena, Nucl. Phys. B472 (1996) 231 [hep-th/9601057]. 
M.R. Garousi and R.C. Myers, Nucl. Phys. B475 (1996) 193 hep-th/9603194.

A. Hashimoto and I.R. Klebanov, Phys. Lett. B381 (1996) 437 hep-th/9604065.

For a review, see: A. Hashimoto and I.R. Klebanov, Nucl. Phys. Proc. Suppl. 55B (1997) 118 [hep-th/9611214].

M.R. Garousi and R.C. Myers, "World-Volume Interaction on D-branes," hepth/9809100.

M.R. Garousi, "Superstring Scattering from D-brane Bound States," hep-th/9805078.

[17] Ming Yu, Bo Zhang, hep-th/9812216, Nucl. Phys. B551 (1999) 425-449.

[18] P. Di Vecchia, M. Frau, I. Pesando, hep-th/9707068, Nucl. Phys. B507 (1997) 259-276.

[19] I. R. Klebanov, A. A. Tseytlin, hep-th/9811035, Nucl. Phys. B546 (1999) 155-181.

Joseph A. Minahan, hep-th/9902074, JHEP 9904 (1999) 007.

[20] Edward Witten, hep-th/9803131, Adv. Theor. Math. Phys. 2 (1998) 505-532. 